\title{
purL gene expression affects biofilm formation and symbiotic persistence of Photorhabdus temperata in the nematode Heterorhabditis bacteriophora
}

\begin{abstract}
Correspondence
Parwinder S. Grewal

grewal.4@osu.edu
\end{abstract}

Received 10 February 2011

Revised 12 May 2011

Accepted 17 June 2011
Ruisheng An and Parwinder S. Grewal

Department of Entomology, Ohio State University, 1680 Madison Ave, Wooster, OH 44691, USA

\begin{abstract}
Extensive studies of the well-known legume and rhizobium symbiosis model system suggest that the purine metabolic pathway plays a key role in microbe-plant interactions, although the exact mechanism is unknown. Here, we report the impact of a key purine metabolic gene, purL, on the symbiotic interaction between the bacterium Photorhabdus temperata and its nematode partner Heterorhabditis bacteriophora. Real-time PCR assays showed that the purL gene was upregulated in $P$. temperata in the nematode infective juvenile compared with artificial media. Mutation of the purL gene by in-frame deletion dramatically decreased the capacity of the bacterium to persist in infective juveniles and its ability to form biofilm in vitro. It was further demonstrated that purL gene expression was positively related to bacterial biofilm formation and the symbiotic persistence of the bacterium in nematode infective juveniles. A $\Delta$ purL mutant lost the ability to support infective juvenile formation in the media which weakly supported biofilm formation, suggesting that a critical level of biofilm formation is required by the bacteria to support infective juvenile formation and thus establish their partnership. In addition, the defects in both biofilm formation and symbiotic ability due to the disruption of the purL gene could be partially restored by the addition of 5 -aminoimidazole-4-carboxamide ribonucleoside (AICAR), an intermediate of the purine biosynthesis pathway. Overall, these data indicate that the purine metabolic pathway is important in microbe-animal symbioses, and that it may influence symbiotic interactions at the level of biofilm formation.
\end{abstract}

\section{INTRODUCTION}

Plant and animal symbioses with micro-organisms are ubiquitous. The characterization of such associations is quintessential in systems biology, as it integrates the study of the interplay between organisms from the different domains of life (McFall-Ngai, 2008). Our understanding of symbioses between microbes and plants has been largely shaped by investigations of Rhizobium bacteria, which are well known for their ability to induce nodules on the roots of leguminous plants, resulting in nitrogen self-sufficiency for the host plant. Bacterial nod genes are accepted as one of the factors required for nodule formation in most rhizobial species. Extensive studies to characterize bacterial auxotrophic mutants have indicated that purine auxotrophy is one of the most universally deleterious symbiotic defects (Giraud et al., 2007; Molloy, 2007). In particular, recent studies in several Rhizobium species have shown that the bacterial purL gene, encoding 5-phosphoribosyl $\mathrm{N}$-formylglycinamide amidotransferase (EC 6.3.5.3), responsible for the conversion of 5-phosphoribosyl $N$-formylglycinamide (FGAR) to 5phosphoribosyl $N$-formylglycinamidine (FGAM) in the purine biosynthesis pathway, is essential for successful

Abbreviation: AICAR, 5-aminoimidazole-4-carboxamide ribonucleoside. nodulation (Buendia-Clavería et al., 2003; Xie et al., 2009). However, the specific role played by the purL gene in nodule formation is still unclear.

Interestingly, our recent results indicate that the purine biosynthesis pathway may also be important to beneficial interactions between microbes and animals (An \& Grewal, 2010). In an emerging animal-microbe symbiosis model system, represented by the partnership between the Gramnegative bacterium Photorhabdus temperata and the entomopathogenic nematode Heterorhabditis bacteriophora (Ruby, 2008), the purL gene has been found to be upregulated by the bacterium during its persistence in nematode infective juveniles (An \& Grewal, 2010). In nature, Photorhabdus bacteria persist in the intestine of the nematode infective juveniles, which endure for months in the soil in search of a suitable insect host (Grewal et al., 2011). The bacteria are released by the infective juveniles into the insect host haemolymph, where they proliferate and produce a plethora of biomolecules, causing rapid insect host mortality (An et al., 2009; Bowen et al., 1998). The bacteria also serve as a food source for the nematodes in the host cadaver, in which nematodes complete one to three generations. The nematodes reproduce by laying eggs either outside or inside the maternal body. The internal 
development of eggs ultimately causes matricide (referred to as endotokia matricida) and produces infective juveniles (Ciche et al., 2008; Johnigk \& Ehlers, 1999). When the insect carcass is depleted of nutrients, the infective juveniles carrying the symbiotic bacteria emerge into the soil in search of a new insect host. As such, the Photorhabdus-Heterorhabditis symbiotic complex serves as a useful biological control agent of diverse insect pests (Grewal et al., 2005).

A fundamental question in the Photorhabdus-Heterorhabditis symbiotic complex as well as other symbioses is how the interactions are initiated, established and maintained. It has been observed that Photorhabdus bacteria infect the intestine of the maternal nematode as a biofilm, and then invade and breach the rectal gland epithelium, becoming available to the developing nematode offspring as they mature into infective juveniles in the pseudocoelom (Ciche et al., 2008; Somvanshi et al., 2010). Studies of Staphylococcus species, which normally inhabit the human oral cavity, suggest that the purL gene and its purine metabolic pathway play an important role in the oral biofilm formation known as dental plaque (Ge et al., 2008; Mack et al., 2004). Therefore, it is reasonable to predict that biofilm formation and purL gene expression may interact to maintain the nematode-bacteria symbiosis and other animal- and plant-microbe symbioses. In this study, we constructed a mutant in the purL gene of $P$. temperata and modified the level of its expression using a complementing plasmid to study the linkage between purL gene expression, biofilm formation and the bacterial ability to interact with the nematode $H$. bacteriophora. Our results demonstrate that purL gene expression can affect both biofilm formation and the symbiotic ability of $P$. temperata, implying that purL-linked biofilm formation may serve as the basis of both plant- and animal-microbe symbiotic interactions.

\section{METHODS}

Strains and growth conditions. Infective juveniles of $H$. bacteriophora GPS11 were obtained from our liquid nitrogen frozen stock and maintained in final instar Galleria mellonella larvae. P. temperata isolated from the infective juveniles was cultured at $25{ }^{\circ} \mathrm{C}$ in Brain Heart Infusion (BHI), Luria-Bertani (LB), nutrient broth or Super Optimal Broth with Catabolite Repression (SOC) medium obtained from BD Diagnostic Systems, with $1.5 \%$ agar added for plates. To determine whether a $\triangle p u r L$ mutant was auxotrophic for purine, the bacteria were also grown in minimal medium (Amos et al., 2011), with adenine $\left(10 \mu \mathrm{g} \mathrm{ml}^{-1}\right)$ and thiamine $\left(1.0 \mu \mathrm{g} \mathrm{ml}^{-1}\right)$ supplementation when required.

Construction of the purL insertion-deletion mutant. An insertion-deletion mutation in the purL gene was constructed using a fusion PCR strategy (Szewczyk et al., 2006). Three fragments, F1 (purL upstream), camR (chloramphenicol-resistance gene) and F2 (purL downstream) were generated using the primer pairs P1 (5'-AAAGGGCCCCACCGTGCGAGATACCCTT-3') and P2 (5' -ACCTCTTACGTGCCGATCAAACCGATGAAGATAATGCGTTGTTA3'), P3 (5'-TTGATCGGCACGTAAGAGGT-3') and P4 (5'-AATTTCTGCCATTCATCCGC-3'), and P5 (5'-GCGGATGAATGGCAGAAATTTCCGGGTTATCGCGTTGAAC-3') and P6 (5' -GCGACTAGTGCCAAGAACAGCAACTTCCGGTCA-3'), respectively. The camR gene was amplified from plasmid pACYC184 (New England BioLabs) and the F1 and F2 fragments from P. temperata genomic DNA. The three fragments were assembled with primers P1 and P6 according to the method described by An et al. (2010). The spliced fragment was restricted and ligated to the ApaI- and SpeI-digested suicide vector pKNG101 (BCCM/LMBP Plasmid Collection, Gent-Zwijnaarde, Belgium). The ligature was conjugated into wild-type $P$. temperata cells via donor Escherichia coli S17-1 $\lambda$ cells. The exconjugants were selected on BHI agar containing $100 \mu \mathrm{g}$ ampicillin $\mathrm{ml}^{-1}$ and $30 \mu \mathrm{g}$ chloramphenicol $\mathrm{ml}^{-1}$ for integration of the ligature into the $P$. temperata chromosome by a single homologous recombination event. The occurrence of a second crossing-over was selected on BHI medium containing $5 \%$ sucrose, resulting in the stably integrated camR gene in the target locus. The phenotype of the created mutant, including bioluminescence, colony morphology and growth rate, was compared with that of the wild-type $P$. temperata in the media described above.

SDS-PAGE analysis of LPS. As purine is usually combined with sugars to participate in the synthesis of the polysaccharide components of cell walls and capsules (Glaser \& Kornfeld, 1961), we also examined whether mutation of the purL gene caused alteration of LPS properties. LPS was isolated from P. temperata wild-type and $\triangle p u r L$ mutant as described by Bennett \& Clarke (2005). Briefly, $3 \mathrm{ml}$ of overnight cultured bacteria grown in BHI medium was washed in $0.9 \% \mathrm{NaCl}$ and centrifuged. The bacterial pellet was resuspended in $100 \mu \mathrm{l}$ buffer containing $50 \mathrm{mM}$ Tris/ $\mathrm{HCl}$ (pH 6.8), $2 \%$ (w/v) SDS and $1 \mathrm{mM}$ EDTA, and was lysed by heating at $100{ }^{\circ} \mathrm{C}$ for $10 \mathrm{~min}$. The lysate was treated with RNase, DNase and proteinase K. The LPS was then separated using a $4-15 \%$ Tris/ $\mathrm{HCl}$ polyacrylamide gel and visualized by the classic silver staining method described by Tsai \& Frasch (1982).

Complementation of the $\Delta$ purL mutant. For complementation, the intact purL gene was amplified from $P$. temperata genomic DNA with primers plu3317F ( $5^{\prime}$-CCCGGTACCCCTTAGGCATCTATTAAGCTTTGC-3') and plu3317R (5'-CCCGAGCTCCTGACATTCCATTAGCCAAAAGG-3'), and was digested with KpnI and SacI restriction enzymes. The promoter region of $r p s M$ amplified from $P$. temperata genomic DNA was cut with SacII and KpnI as described elsewhere (Münch et al., 2008), and fused with the digested purL gene. The fusion product was cloned into the enzyme-treated pJB861 vector, resulting in plasmid pJB861-purL. Both the pJB861 vector and the pJB861-purL plasmid were introduced into P. temperata wild-type and $\Delta$ purL mutant via electroporation, as described by Jamoussi et al. (2009) for complementation analysis. The rpsM promoter was chosen for trans expression of the purL gene, based on the consideration that earlier studies of Salmonella bacteria have suggested that the genes under its control are constitutively expressed at similar levels in various environments, including growth media and mammalian cells (Hautefort et al., 2003; Valdivia \& Falkow, 1997).

Symbiosis assays. The effects of the purL mutation on bacterial symbiotic ability to support nematode development and persist in infective juveniles were assessed. Overnight cultured $P$. temperata wild-type and $\Delta$ purL mutant cells $(50 \mu \mathrm{l})$ were independently spread on cholesterol agar plates $\left(1.5 \%\right.$ agar and $10 \mu \mathrm{g}$ cholesterol $\left.\mathrm{ml}^{-1}\right)$ containing the tested media and incubated at $25{ }^{\circ} \mathrm{C}$ for $24 \mathrm{~h}$. When required, the media were supplemented with $10 \mu \mathrm{g}$ adenine $\mathrm{ml}^{-1}$ and/or $50 \mu \mathrm{g} \mathrm{ml}^{-1}$ 5-aminoimidazole-4-carboxamide ribonucleoside (AICAR). Approximately 100 bacteria-free H. bacteriophora eggs obtained from hermaphrodites using an alkaline lysis method (Lunau et al., 1993) were propagated on each plate at $25^{\circ} \mathrm{C}$. Production of infective juveniles was monitored daily under a microscope. The freshly produced infective juveniles were harvested from the plate and stored in water with or without supplementation of adenine and/or AICAR, as appropriate. The numbers of bacterial cells in freshly produced and 30 day-old infective juveniles were then assessed using 
the method described previously (An \& Grewal, 2010). As maternal adhesion of the bacteria is essential for their transmission to the infective juvenile stage offspring developing inside the maternal body (Ciche et al., 2008; Somvanshi et al., 2010), we also determined whether the $\Delta$ purL mutant was defective in its ability to persist in the maternal intestine. Adult females were removed from the bacterial lawn and starved in sterile water for 4 and $24 \mathrm{~h}$, during which time the water was changed periodically. The starved nematodes were thoroughly washed with $75 \%$ alcohol and sterile water, and then homogenized to detect persistent colonizing cells. All assays were performed in triplicate using three independent cultures of the test bacterium. The data were subjected to one-way analysis of variance with significant differences determined at $P=0.05$.

Biofilm formation assay. Biofilm formation was assayed as the ability of cells to adhere to the wells of polystyrene microplates, as described elsewhere (O’Toole \& Kolter, 1998). The medium under test $(100 \mu \mathrm{l})$ in each well was inoculated with $1 \mu \mathrm{l}$ of overnight cultured $P$. temperata cells. For anaerobic growth, the media were boiled first to drive off oxygen, covered with $50 \mu$ sterilized mineral oil, cooled down for $30 \mathrm{~min}$, and then inoculated with overnight cultured bacterial cells. After incubation at $28{ }^{\circ} \mathrm{C}$ for $24 \mathrm{~h}$, bacterial growth was determined with a plate reader at $450 \mathrm{~nm}$. Then, $25 \mu \mathrm{l}$ $1 \%(\mathrm{w} / \mathrm{v})$ crystal violet was added to each well, incubated at room temperature for $15 \mathrm{~min}$, and rinsed away by three washes with $200 \mu \mathrm{l}$ distilled $\mathrm{H}_{2} \mathrm{O}$. Biofilm formation was quantified by the addition of $200 \mu \mathrm{l} 95 \%$ ethanol to each crystal violet-stained well, followed by measurement of the $A_{600}$ with a plate reader. The assays were performed in triplicate, and the significant differences for biofilm formation were tested by one-way analysis of variance with a confidence level of $95 \%$.

Quantitative real-time PCR. The expression of the bacterial purL gene in culture media and freshly produced infective juveniles was quantified by real-time PCR in an IQ5 system (Bio-Rad) using a QuantiTect SYBR Green PCR kit (Qiagen) according to the manufacturer's instructions. All reactions were run in triplicate with three independent RNA samples and a no-template negative control using the primer pair purLF (5'-AATGCGGCGGCCATTTCTACATGA-3') and purLR (5'-GCATCAGGCCAAACGGGATAATCA-3'). The relative fold change was calculated from the $\Delta \Delta C_{\mathrm{t}}$ by using two internal control genes, 16S rRNA and the prokaryotic housekeeping gene gyrA (An \& Grewal, 2010; Daborn et al., 2001), as described by Livak \& Schmittgen (2001).

\section{RESULTS}

\section{Fitness of the $P$. temperata $\Delta$ purL mutant}

To determine the role of purL in bacterial association with the nematode partner, a $P$. temperata $\Delta p u r L$ in-frame deletion mutant was created through insertion-deletion mutation and allelic exchange. The deletion fragment containing $1041 \mathrm{bp}$ from positions 1428 to 2463 of the purL ORF was replaced by the complete chloramphenicolresistance gene $\operatorname{cam} R$. The success of gene disruption and the stability of the replacement in the created mutant were confirmed by several rounds of subculture, followed by sequence analyses of the PCR product in the purL region. In BHI, LB, nutrient broth and SOC media, the mutant was similar to the wild-type in general phenotypic properties, including bioluminescence, colony morphology and growth rate, and both the wild-type and the $\Delta p u r L$ mutant were still viable after 10 days in all of the media. Unlike wild-type $P$. temperata cells, however, the $\Delta$ purL mutant did not grow in minimal medium unless it was supplemented with both adenine and thiamine, suggesting that the mutant is auxotrophic for purine.

\section{LPS profile}

SDS-PAGE analysis of $P$. temperata cells grown in BHI medium detected no alteration in the LPS profile with the insertion-deletion mutation of the purL gene (Fig. 1). Earlier studies of rhizobia have reported that the LPS bands of a purL mutant created through transposon mutagenesis migrate faster than those of the parental strain (BuendiaClavería et al., 2003), although no difference was detected between the wild-type and a purL mutant constructed through insertion-deletion mutation (Xie et al., 2006). While we do not rule out the possibility that a change in LPS was occurring but could not be detected via SDSPAGE, these results may otherwise suggest that some portion of the upstream, but not downstream, region of the purL gene may be associated with the LPS phenotype change. Alternatively, the LPS biosynthesis pathway in Photorhabdus may be divergent from those of rhizobia.

\section{Effect of purL mutation on bacterial ability to support nematode reproduction}

The mutation of the P. temperata purL gene did not affect the egg hatch of $H$. bacteriophora. Most of the eggs hatched within $24 \mathrm{~h}$ post-propagation on the bacterial cells grown

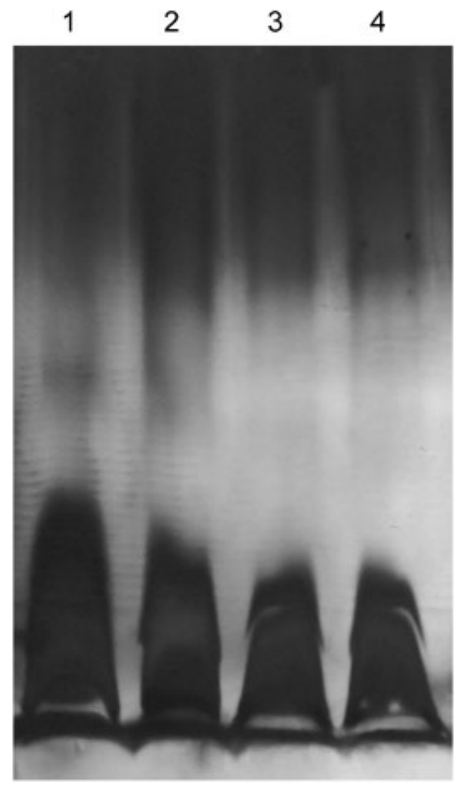

Fig. 1. Silver stained SDS-PAGE showing LPS profiles of bacterial cells grown in $\mathrm{BHI}$ medium. Lanes: 1, E. coli strain SM10; 2, Photorhabdus luminescens strain TT01; 3, wild-type P. temperata strain GPS $11 ; 4, P$. temperata $\Delta$ purL mutant. 
in the tested media, including BHI, LB, nutrient broth and SOC. Nematode reproduction was well supported by both the wild-type and the $\Delta$ purL mutant in BHI, LB and SOC media, despite slightly lower IJ production from the $\Delta p u r L$ strain relative to the wild-type when grown in BHI and $\mathrm{LB}$ media (Fig. 2). However, when grown in nutrient broth medium, nematode reproduction was barely supported by the mutant cells. Infective juveniles were not produced on the $\Delta$ purL mutant and there were only a few adults present on the nutrient broth medium plates after 10 days. Interestingly, for nematodes grown on BHI, LB and SOC media, both the wild-type and the mutant cells could be detected in the adult females after starvation for 4 and $24 \mathrm{~h}$; however, when grown on nutrient broth medium, the mutant cells were detected only after $4 \mathrm{~h}$ and not after $24 \mathrm{~h}$ starvation. Also, on nutrient broth medium, although the eggs were initially laid and hatched in the maternal body, further development did not occur (Fig. 3). Complementation with the pJB861-purL plasmid restored the mutant to the wild-type level in supporting nematode reproduction in nutrient broth medium (Fig. 2). Supplementation with adenine had no effect on the $\Delta p u r L$ mutant in supporting nematode reproduction in nutrient broth medium, whereas the addition of AICAR partially restored the ability of the mutant to support nematode reproduction. The number of infective juveniles yielded on the $\Delta$ purL mutant in the presence of AICAR was about $20 \%$ of those formed on either the wild-type or the mutant carrying the pJB861purL plasmid (Fig. 2).

\section{Effect of purL mutation on bacterial colonization and persistence in the infective juveniles}

The level of infective juvenile colonization achieved by the bacteria was essentially the same in all the freshly produced infective juveniles, but decreased dramatically 30 days later in the case of the $\Delta$ purL mutant (Fig. 4). Infective juveniles produced on the wild-type retained significantly $(P<0.01)$ more bacterial cells after 30 days compared with those produced on the mutant cells grown in either LB or nutrient broth medium supplemented with AICAR, suggesting that the purL gene is critical for bacterial
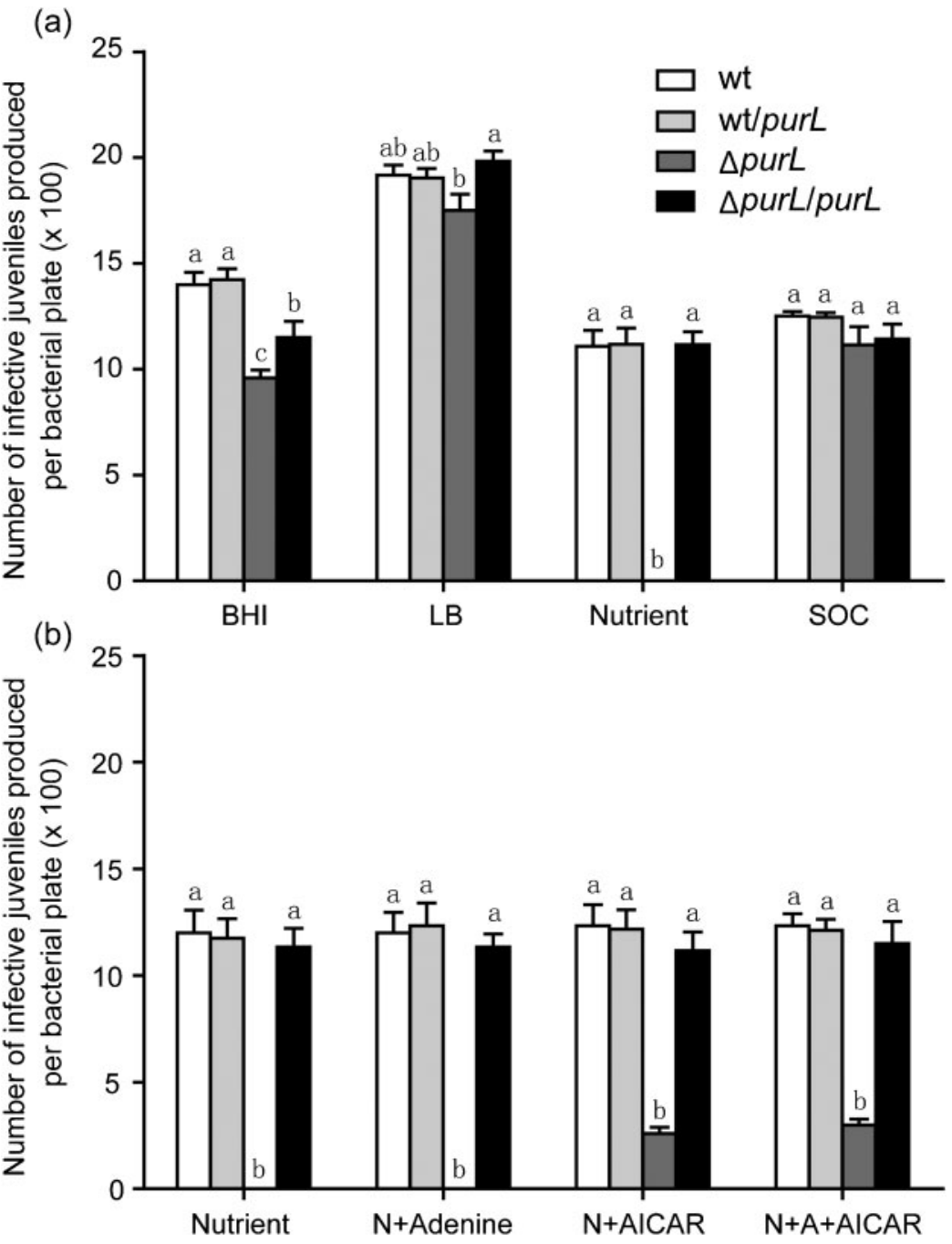

Fig. 2. Production of $H$. bacteriophora infective juveniles with $P$. temperata cells grown in different media. (a) Bacteria were grown on $\mathrm{BHI}, \mathrm{LB}$, nutrient broth and SOC media; (b) bacteria were grown on nutrient broth medium (N) supplemented with $10 \mu \mathrm{g}$ adenine $\mathrm{ml}^{-1}$ (A) and/or $50 \mu \mathrm{g} \mathrm{AICAR} \mathrm{ml}^{-1}$. The data are the mean number ( $\pm \mathrm{SEM}, n=3$ ) of $H$. bacteriophora infective juveniles produced 10 days after inoculation of 100 bacteria-free nematode eggs on plates seeded with wild-type (wt) bacteria, wild-type carrying the purL expression plasmid (wt/purL), spurL mutant $(\Delta p u r L)$ or the mutant complemented with the purL-containing plasmid ( $\Delta$ purL/purL). Significant differences among different bacterial cell types in each medium are indicated by different letters. 

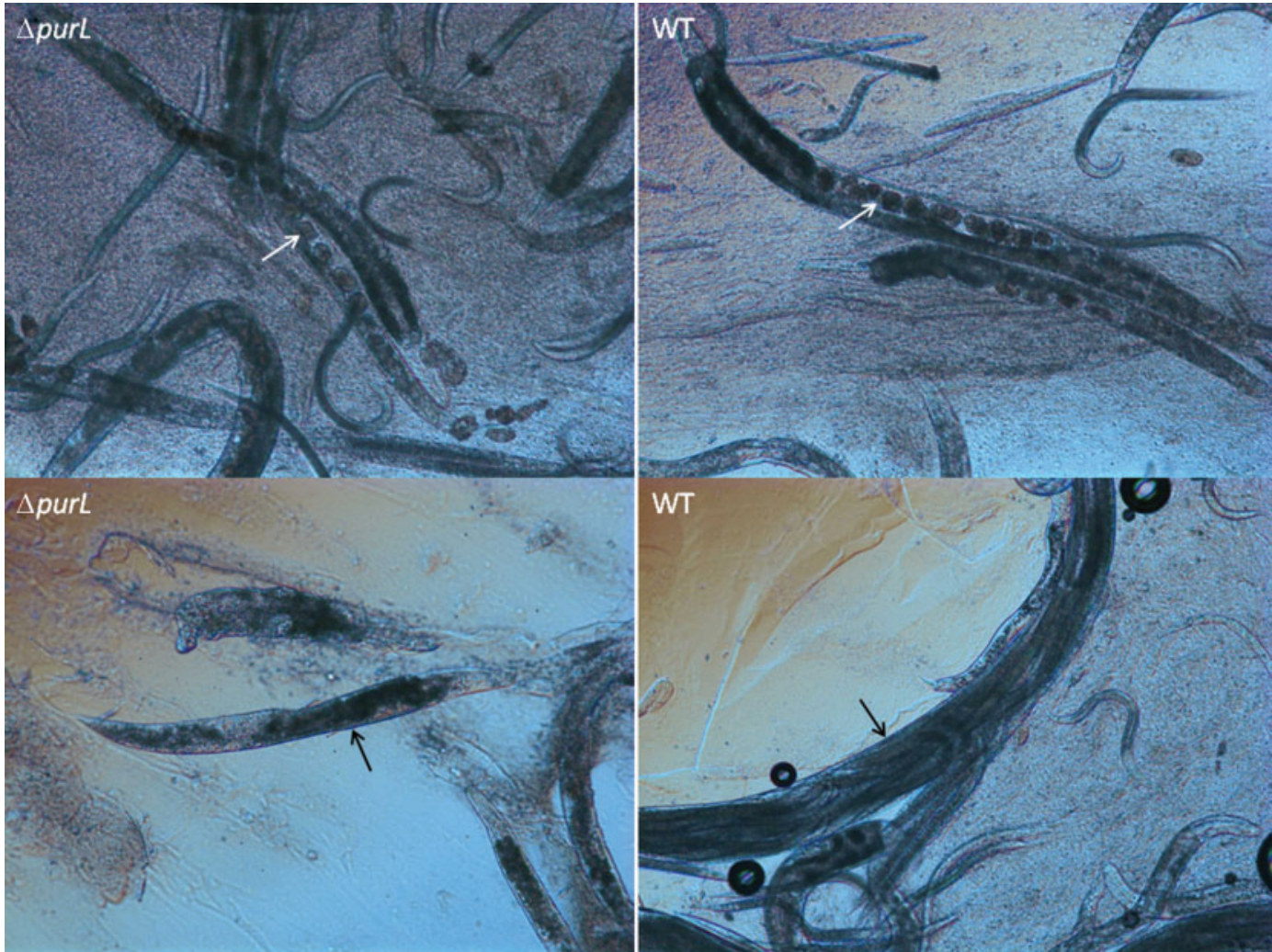

Fig. 3. Microscopic observation of $H$. bacteriophora development with $P$. temperata wild-type (WT) and the $\Delta$ purL mutant grown in nutrient broth agar. Eggs (white arrows) were initially laid and hatched internally, but their development into infective juveniles (black arrows) ceased in the maternal body when cultured on a lawn of mutant bacteria.

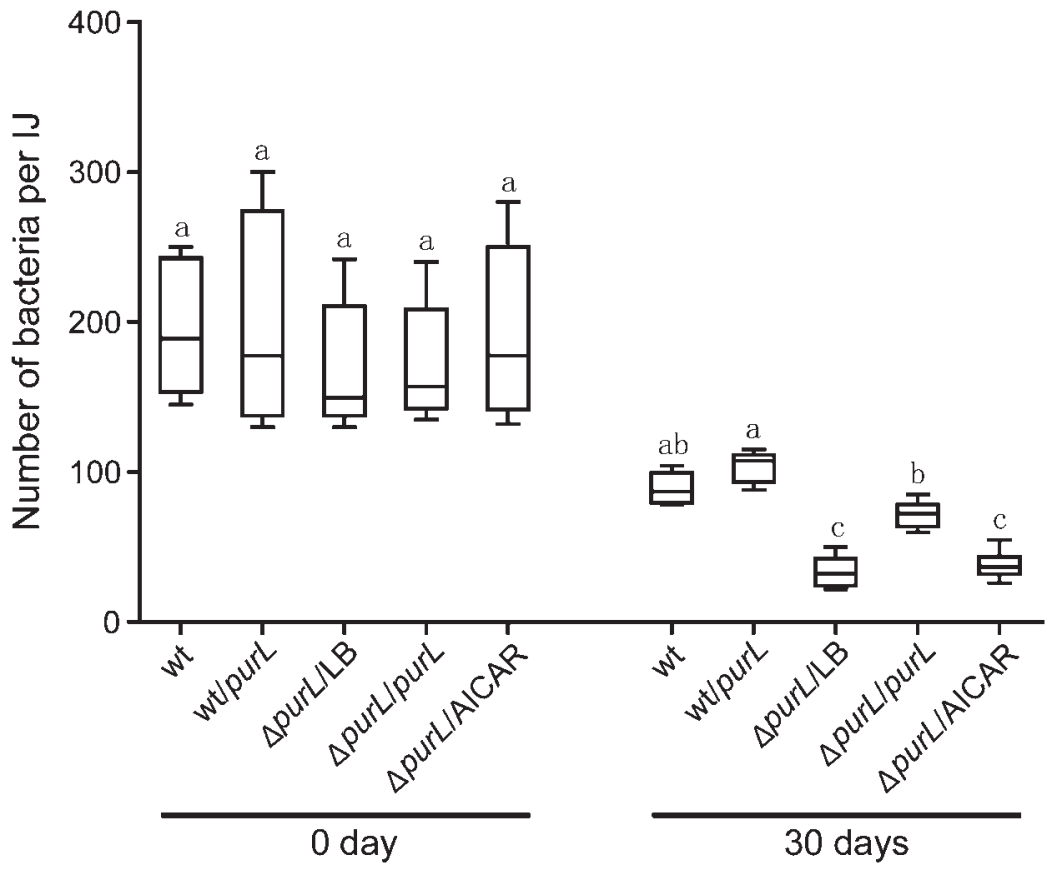

Fig. 4. Persistence of $P$. temperata in the H. bacteriophora infective juvenile. The infective juveniles (IJs) were produced on $P$. temperata wild-type cells grown in nutrient broth medium (wt), wild-type cells containing the purL-expression plasmid grown in nutrient broth medium (wt/purL), the $\Delta$ purL mutant grown in LB medium ( $\Delta$ purL/LB), the $\Delta$ purL mutant complemented with the purL-expression plasmid grown in nutrient broth medium ( $\Delta$ purL/purL), and the $\Delta$ purL mutant grown in nutrient broth medium supplemented with $50 \mu \mathrm{g} \mathrm{AICAR} \mathrm{\textrm {ml } ^ { - 1 }}$ ( $\triangle$ purL/AICAR). The numbers of $P$. temperata cells in freshly produced (0 day) and 30 dayold $H$. bacteriophora IJs are presented using box and whisker plots which show the median value (horizontal line), the upper and lower quartiles (boxed) and the range of the data (whiskers). Significant differences among different bacterial cell types at each time point are indicated by different letters. 
symbiotic persistence. Complementation of the mutant with the purL-carrying plasmid resulted in the restoration of persistence almost to the wild-type level, further confirming the importance of this gene in bacterial symbiotic persistence in the nematode. In addition, unlike the purL-carrying plasmid, the presence of AICAR and/or adenine in water had no effect on the persistence of the bacteria in 30 day-old infective juveniles. This demonstrates that the rescue ability of AICAR for the persistence of bacteria in the infective juvenile differs from that for either the maternal persistence or the biofilm phenotype.

\section{Effect of purL mutation on bacterial biofilm formation}

As biofilm is likely to be important in the PhotorhabdusHeterorhabditis interaction (Ciche et al., 2008; Somvanshi et al., 2010), we further examined the impact of purL disruption on biofilm formation in $P$. temperata. The mutation of the purL gene resulted in a 30-60\% reduction in biofilm formation in the four tested media, and trans expression of the purL gene via the transformed plasmid restored the biofilm formation phenotype (Fig. 5). While this result suggested that the purL gene was responsible for biofilm formation regardless of the specific medium used, the mutant formed the weakest biofilm in nutrient broth medium as measured by crystal violet absorption at $600 \mathrm{~nm}$. In BHI, LB and SOC media, the $\triangle$ purL mutant retained a much higher biofilm formation capacity that was similar to that of wild-type cells in nutrient broth medium. Further, while supplementation with adenine had no effect on the biofilm formation of the $\Delta$ purL mutant in nutrient broth medium, the impaired biofilm formation in the mutant cells could be partially restored by the presence of AICAR (Fig. 6), suggesting that the purL gene and its metabolic pathway are essential to biofilm formation in P. temperata.

\section{Real-time PCR analysis of purL gene expression}

A quantitative real-time PCR assay detected about an eightfold increase in purL expression in P. temperata in the nematode infective juveniles compared with the tested
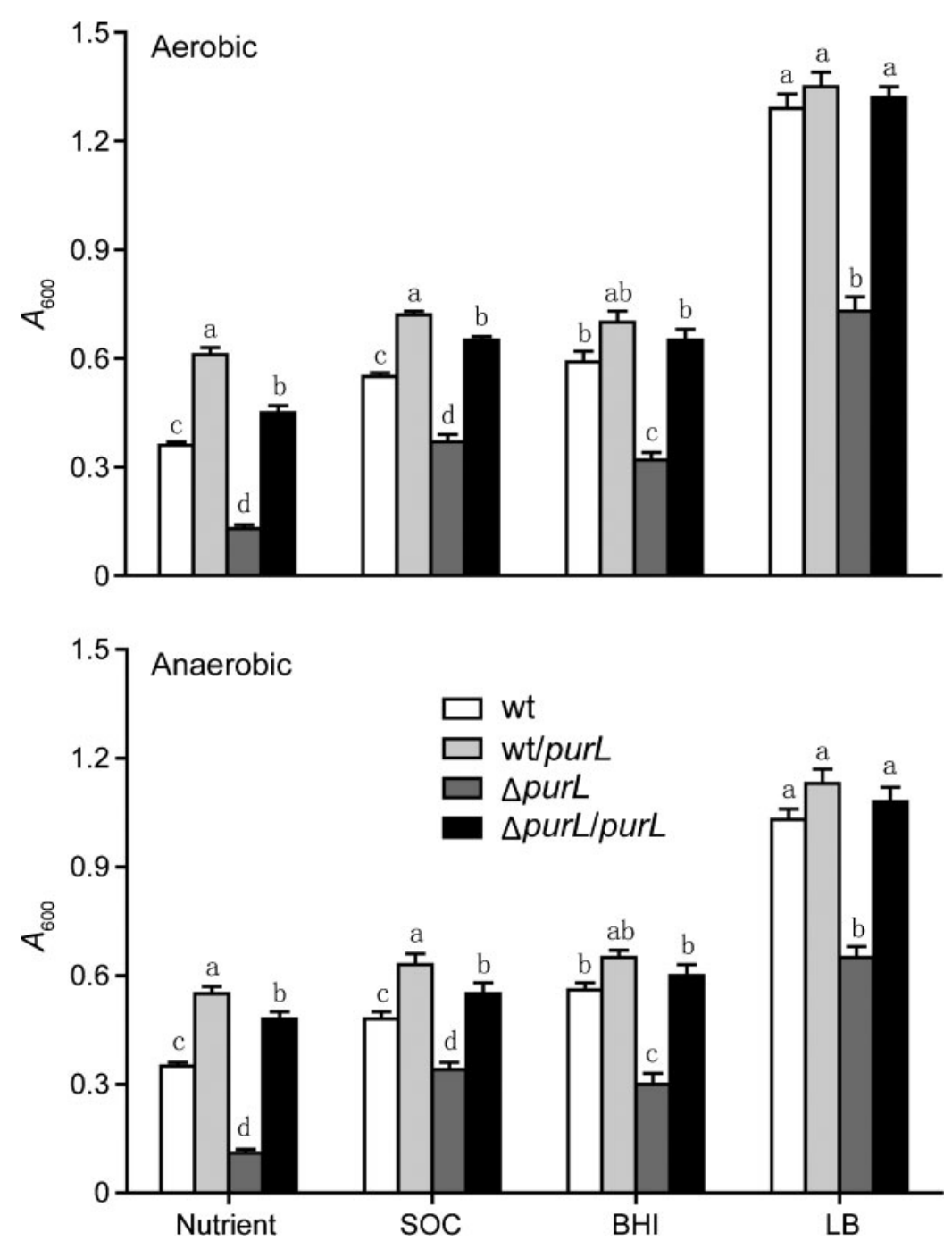

Fig. 5. $P$. temperata biofilm formation in different media, $\mathrm{LB}, \mathrm{BHI}$ and SOC. The data are mean values ( $\pm \mathrm{SEM}, n=3$ ) representing the level of biofilm formation as measured via crystal violet absorption at $600 \mathrm{~nm}$ after $24 \mathrm{~h}$ incubation under aerobic and anaerobic conditions. Significant differences among different bacterial cell types in each medium are marked with different letters. 

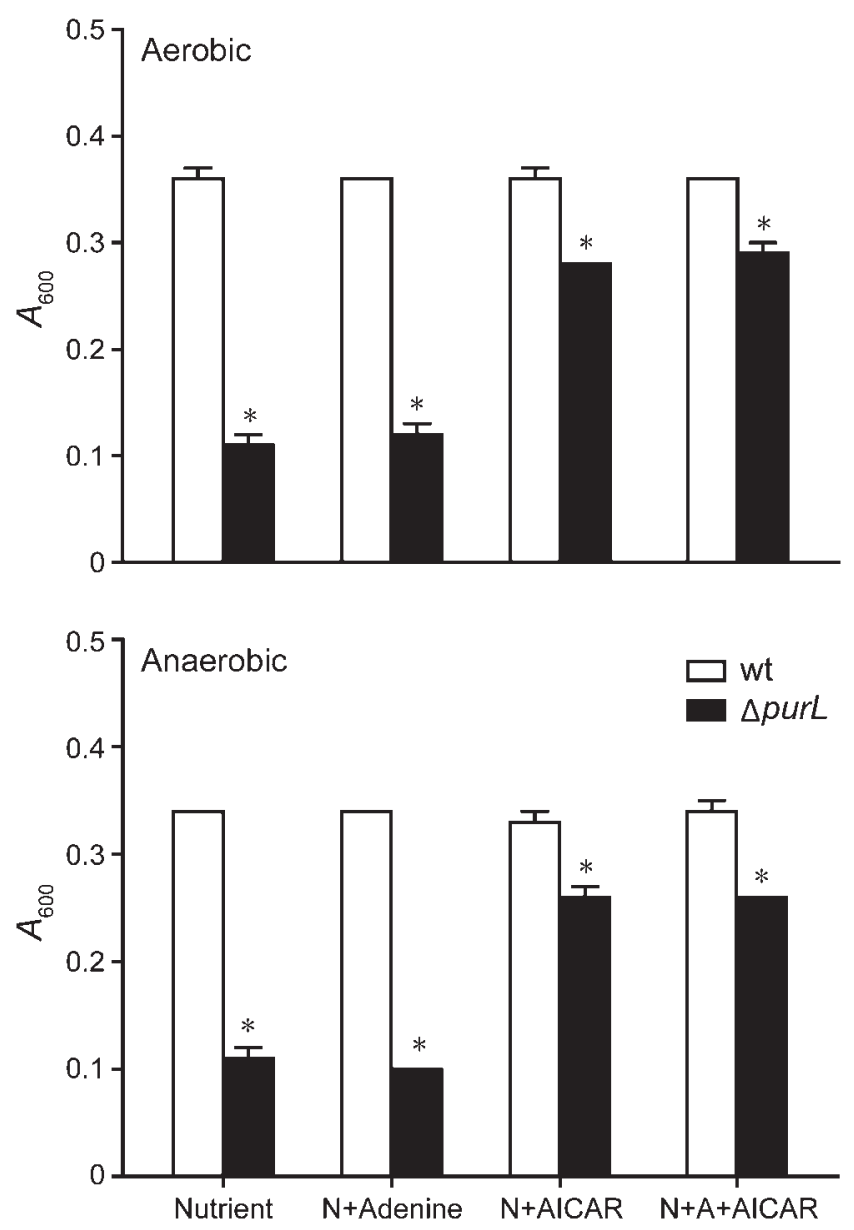

Fig. 6. Biofilm formation of $P$. temperata wild-type and $\Delta$ purL mutant cells. Bacteria were grown in the indicated media: nutrient broth, nutrient broth supplemented with $10 \mu \mathrm{g}$ adenine $\mathrm{ml}^{-1}$ ( $\mathrm{N}+$ Adenine), nutrient broth with $50 \mu \mathrm{g} \mathrm{AICAR} \mathrm{\textrm {m } ^ { - 1 }}(\mathrm{N}+\mathrm{AICAR})$, and nutrient broth with both AICAR and adenine ( $+A+A I C A R)$. The data are mean values ( $\pm \mathrm{SEM}, n=3$ ) representing the level of biofilm formation as measured via crystal violet absorption at $600 \mathrm{~nm}$ after $24 \mathrm{~h}$ incubation under aerobic and anaerobic conditions. Significant differences between the wild-type and the mutant in each tested medium are indicated by asterisks.

media in vitro. The constitutive trans expression of purL in the mutant under the control of the housekeeping $r p s M$ promoter on the constructed plasmid was about fourfold greater than chromosomal expression in the wild-type grown in vitro. Similarly, the expression of the purL gene in the wild-type during persistence in the infective juveniles increased threefold upon receiving the constructed plasmid. These data confirm that the purL gene plays an important role in bacterial persistence in the infective juveniles.

\section{DISCUSSION}

Microbial symbioses with animals and plants are ubiquitous, and characterization of the patterns of their associations in a diverse set of model systems promises to revolutionize the way we view the biotic world (McFall-Ngai, 2008). The symbiosis between Photorhabdus bacteria and Heterorhabditis nematodes represents one of the best-developed microbe-animal symbiosis models (Clarke, 2008; Ruby, 2008). Our results indicate that the expression of the purL gene involved in the purine metabolic pathway is important for the colonization and persistence of $P$. temperata in the infective juveniles of $H$. bacteriophora. Interestingly, a critical role for the purL gene in the rhizobium-legume interaction, a well-known microbe-plant symbiosis model, has also been suggested, as nodulation failures of purL mutants have been described in several rhizobial species, including those of the genera Sinorhizobium and Bradyrhizobium (Giraud et al., 2007; Xie et al., 2006). Therefore, the present discovery suggests a more universal role for the purine metabolic pathway in beneficial microbial symbioses.

During the Heterorhabditis-Photorhabdus symbiotic interaction, the nematodes use bacteria as a food source, while the bacterium uses the nematode as a vector to reach its final destination, the insect host. Our results show that the $\Delta$ purL mutant supports nematode reproduction in BHI, LB and SOC media but not in nutrient broth medium, which could be explained by the possibility that the first three media contain purine pathway derivatives. While purL gene expression is required to support nematode reproduction and infective juvenile formation conditionally depending on the growth medium, it is always necessary for the bacteria to persist in the nematode infective juvenile. Further, the ability of the bacteria to persist in the infective juveniles appears to be correlated with the level of purL expression. Constitutive trans expression of the purL gene through the transformed plasmid enabled the wild-type cells to persist much better than the mutant cells. Unlike purL gene complementation, additional AICAR had no effect on the persistence of the bacteria in the infective juveniles, although it partially rescued the mutant defect in supporting infective juvenile production and biofilm formation. Previous observations reveal that the nematode infective juvenile is a closed system, thus preventing penetration by microbial antagonists and toxic chemicals (Glazer, 2002; Mracek et al., 1981). Therefore, one explanation for the inability of AICAR to rescue the persistence defect could be the possibility that once the infective juvenile forms, AICAR most likely cannot be transported into the area that houses the bacteria.

As a mode of life, biofilm is often crucial for survival of bacteria under environmental stresses such as nutrient limitation and desiccation (Rinaudi \& Giordano, 2010). We found that $P$. temperata purL gene expression is positively related to biofilm formation, which is also influenced by the growth medium. For the bacteria grown in vitro, although biofilm formation was significantly impaired in all the tested media due to the purL gene mutation, a higher level of biofilm formation was observed in BHI, LB and SOC media compared with nutrient broth medium. Unlike growth in culture media, bacteria in 
non-feeding infective juveniles persist under nutrient limitation conditions (An \& Grewal, 2010), and consequently their biofilm formation may mainly rely on the upregulation of purL gene expression, which is indeed confirmed by our real-time PCR assays. Therefore, we conclude that purL gene-regulated biofilm formation may play an essential role in bacterial persistence in the nematode infective juvenile.

An earlier study suggests that Photorhabdus bacteria infect the intestine of the adult nematode via biofilm becoming available for the offspring to develop into infective juveniles in the maternal body cavity (Ciche et al., 2008). Our results suggest that a critical level of biofilm formation may be required by the bacteria to successfully infect the intestine of the adult nematode. In this study, disruption of the purL gene resulted in the weakest biofilm formation in nutrient broth medium, and correspondingly the mutant was unable to persist in the maternal intestine and become available to developing infective juveniles when grown in this medium. The nematode eggs were initially laid but failed to develop in the maternal body when propagated on the $\Delta$ purL mutant in nutrient broth medium, suggesting that the bacteria with poor biofilm formation capacity may be incapable of breaching the nematode rectal gland epithelium to reach the hatched eggs in the maternal body. Along with the partially increased biofilm formation induced by the addition of AICAR to nutrient broth medium, the ability of the $\triangle$ purL mutant to support infective juvenile formation was enhanced. Together, these data suggest that a threshold level of biofilm formation is critical for the bacteria to support infective juvenile formation and thus establish their symbiotic partnership.

Our findings on the linkage among purL gene expression, biofilm formation and bacterial symbiotic properties also provide a perspective for studies in other symbiotic systems. In the rhizobium-legume symbiosis, previous studies have indicated that the biofilm lifestyle allows rhizobia to survive under unfavourable conditions, although it remains unclear whether and how rhizobial biofilm formation affects the degree of symbiosis with the legume host (Rinaudi \& Giordano, 2010). In this study, we have shown that biofilm formation is positively correlated with the level of purL gene expression, which also significantly affects the persistence of the bacterial symbiont in its nematode partner. As purL gene expression has also been suggested to be important in the rhizobium-legume symbiosis, future research may focus on determining how the rhizobial biofilm is integrated with nodulation in the roots of legume plants through regulation of purL gene expression.

Finally, as AICAR, but not adenine, could only partially rescue the defective ability of the $\Delta$ purL mutant in supporting infective juvenile production and biofilm formation, we speculate that besides the purine pathway this gene may also have other effects which could not be rescued by AICAR. In fact, purL is well known in E. coli as a fused gene, being composed of three different domains, one of which functions as a glutaminase, acting in histidine metabolism to generate AICAR and glutamate (Sampei \& Mizobuchi, 1989). Future research on the whole purine metabolic pathway and its impact on biofilm formation may enable us to gain a better understanding of bacterial symbioses with plants and animals.

\section{ACKNOWLEDGEMENTS}

This study was supported by a competitive grant from the Ohio Agricultural Research and Development Center, The Ohio State University, Wooster, $\mathrm{OH}$.

\section{REFERENCES}

Amos, M. R., Sanchez-Contreras, M., Jackson, R. W., Muñoz-Berbel, X., Ciche, T. A., Yang, G., Cooper, R. M. \& Waterfield, N. R. (2011). Influence of the Photorhabdus luminescens phosphomannose isomerase gene, $m a n A$, on mannose utilization, exopolysaccharide structure, and biofilm formation. Appl Environ Microbiol 77, 776-785.

An, R. \& Grewal, P. S. (2010). Molecular mechanisms of persistence of mutualistic bacteria Photorhabdus in the entomopathogenic nematode host. PLoS ONE 5, e13154.

An, R., Sreevatsan, S. \& Grewal, P. S. (2009). Comparative in vivo gene expression of the closely related bacteria Photorhabdus temperata and Xenorhabdus koppenhoeferi upon infection of the same insect host, Rhizotrogus majalis. BMC Genomics 10, 433.

An, R., Bai, X. \& Grewal, P. S. (2010). Reliable fusion PCR using Taq polymerases and pGEM-T easy vectors. World J Microbiol Biotechnol 27, 727-730.

Bennett, H. P. \& Clarke, D. J. (2005). The pbgPE operon in Photorhabdus luminescens is required for pathogenicity and symbiosis. J Bacteriol 187, 77-84.

Bowen, D., Rocheleau, T. A., Blackburn, M., Andreev, O., Golubeva, E., Bhartia, R. \& ffrench-Constant, R. H. (1998). Insecticidal toxins from the bacterium Photorhabdus luminescens. Science 280, 2129-2132.

Buendia-Clavería, A. M., Moussaid, A., Ollero, F. J., Vinardell, J. M., Torres, A., Moreno, J., Gil-Serrano, A. M., Rodríguez-Carvajal, M. A., Tejero-Mateo, P. \& other authors (2003). A purL mutant of Sinorhizobium fredii $\mathrm{HH} 103$ is symbiotically defective and altered in its lipopolysaccharide. Microbiology 149, 1807-1818.

Ciche, T. A., Kim, K. S., Kaufmann-Daszczuk, B., Nguyen, K. C. Q. \& Hall, D. H. (2008). Cell invasion and matricide during Photorhabdus luminescens transmission by Heterorhabditis bacteriophora nematodes. Appl Environ Microbiol 74, 2275-2287.

Clarke, D. J. (2008). Photorhabdus: a model for the analysis of pathogenicity and mutualism. Cell Microbiol 10, 2159-2167.

Daborn, P. J., Waterfield, N., Blight, M. A. \& ffrench-Constant, R. H. (2001). Measuring virulence factor expression by the pathogenic bacterium Photorhabdus luminescens in culture and during insect infection. J Bacteriol 183, 5834-5839.

Ge, X. C., Kitten, T., Chen, Z. M., Lee, S. P., Munro, C. L. \& Xu, P. (2008). Identification of Streptococcus sanguinis genes required for biofilm formation and examination of their role in endocarditis virulence. Infect Immun 76, 2551-2559.

Giraud, E., Moulin, L., Vallenet, D., Barbe, V., Cytryn, E., Avarre, J. C., Jaubert, M., Simon, D., Cartieaux, F. \& other authors (2007). Legumes symbioses: absence of nod genes in photosynthetic bradyrhizobia. Science 316, 1307-1312. 
Glaser, L. \& Kornfeld, S. (1961). The enzymatic synthesis of thymidine-linked sugars. II. Thymidine diphosphate L-rhamnose. J Biol Chem 236, 1795-1799.

Glazer, I. (2002). Survival biology. In Entomopathogenic Nematology, pp. 169-187. Edited by R. Gaugler. New York: CABI Publishing.

Grewal, P. S., Ehlers, R. U. \& Shapiro-Ilan, D. I. (2005). Nematodes as Biocontrol Agents. Wallingford, UK: CABI Publishing.

Grewal, P. S., Bai, X. \& Jagdale, G. B. (2011). Longevity and stress tolerance of entomopathogenic nematodes. In Molecular and Physiological Basis of Nematode Survival, pp. 157-181. Edited by R. N. Perry \& D. Wharton. Wallingford, UK: CABI Publishing.

Hautefort, I., Proença, M. J. \& Hinton, J. C. (2003). Single-copy green fluorescent protein gene fusions allow accurate measurement of Salmonella gene expression in vitro and during infection of mammalian cells. Appl Environ Microbiol 69, 7480-7491.

Jamoussi, K., Sellami, S., Abdelkefi-Mesrati, L., Givaudan, A. \& Jaoua, S. (2009). Heterologous expression of Bacillus thuringiensis vegetative insecticidal protein-encoding gene vip3LB in Photorhabdus temperata strain $\mathrm{K} 122$ and oral toxicity against the lepidoptera Ephestia kuehniella and Spodoptera littoralis. Mol Biotechnol 43, 97103.

Johnigk, S. A. \& Ehlers, R. U. (1999). Endotokia matricida in hermaphrodites of Heterorhabditis spp. and the effect of the food supply. Nematology 1, 717-726.

Livak, K. J. \& Schmittgen, T. D. (2001). Analysis of relative gene expression data using real-time quantitative PCR and the $2^{-\Delta \Delta C_{\mathrm{T}}}$ method. Methods 25, 402-408.

Lunau, S., Stoessel, S., Schmidtpeisker, A. J. \& Ehlers, R. U. (1993). Establishment of monoxenic inocula for scaling-up in-vitro cultures of the entomopathogenic nematodes Steinernema spp. and Heterorhabditis spp. Nematologica 39, 385-399.

Mack, D., Becker, P., Chatterjee, I., Dobinsky, S., Knobloch, J. K. M., Peters, G., Rohde, H. \& Herrmann, M. (2004). Mechanisms of biofilm formation in Staphylococcus epidermidis and Staphylococcus aureus: functional molecules, regulatory circuits, and adaptive responses. Int J Med Microbiol 294, 203-212.

McFall-Ngai, M. (2008). Are biologists in 'future shock'? Symbiosis integrates biology across domains. Nat Rev Microbiol 6, 789-792.
Molloy, S. (2007). Symbiotic exceptions. Nat Rev Microbiol 5, 473.

Mracek, Z., Gerdin, S. \& Weiser, J. (1981). Head and cuticular structure of some species in the family Steinernematidae (Nematoda). Nematologica 27, 443-448.

Münch, A., Stingl, L., Jung, K. \& Heermann, R. (2008). Photorhabdus luminescens genes induced upon insect infection. BMC Genomics $\mathbf{9}$, 229.

O'Toole, G. A. \& Kolter, R. (1998). Flagellar and twitching motility are necessary for Pseudomonas aeruginosa biofilm development. Mol Microbiol 30, 295-304.

Rinaudi, L. V. \& Giordano, W. (2010). An integrated view of biofilm formation in rhizobia. FEMS Microbiol Lett 304, 1-11.

Ruby, E. G. (2008). Symbiotic conversations are revealed under genetic interrogation. Nat Rev Microbiol 6, 752-762.

Sampei, G. \& Mizobuchi, K. (1989). The organization of the purL gene encoding 5 '-phosphoribosylformylglycinamide amidotransferase of Escherichia coli. J Biol Chem 264, 21230-21238.

Somvanshi, V. S., Kaufmann-Daszczuk, B., Kim, K. S., Mallon, S. \& Ciche, T. A. (2010). Photorhabdus phase variants express a novel fimbrial locus, mad, essential for symbiosis. Mol Microbiol 77, 1021-1038.

Szewczyk, E., Nayak, T., Oakley, C. E., Edgerton, H., Xiong, Y., Taheri-Talesh, N., Osmani, S. A. \& Oakley, B. R. (2006). Fusion PCR and gene targeting in Aspergillus nidulans. Nat Protoc 1, 3111-3120.

Tsai, C. M. \& Frasch, C. E. (1982). A sensitive silver stain for detecting lipopolysaccharides in polyacrylamide gels. Anal Biochem 119, 115119.

Valdivia, R. H. \& Falkow, S. (1997). Fluorescence-based isolation of bacterial genes expressed within host cells. Science 277, 2007-2011.

Xie, B., Chen, D. S., Zhou, K., Xie, Y. Q., Li, Y. G., Hu, G. Y. \& Zhou, J. C. (2006). Symbiotic abilities of Sinorhizobium fredii with modified expression of purL. Appl Microbiol Biotechnol 71, 505-514.

Xie, B., Chen, D. S., Cheng, G. J., Ying, Z. Z., Xie, F. L., Li, Y. G. \& Zhou, J. C. (2009). Effects of the purL gene expression level on the competitive nodulation ability of Sinorhizobium fredii. Curr Microbiol 59, 193-198.

Edited by: L. Knoll 